\title{
Efecto de los nidos de ACROMYRMEX LOBICORNIS (FoRMICIDAE: MYRMICINAE) SOBRE LA DESCOMPOSICIÓN DE HOJARASCA, EN EL DESIERTO DEL MONTE
}

\author{
ANA LAURA NAVAS ROMERO ${ }^{1 *}$, MARIO ANDRÉS HERRERA MORATTA ${ }^{1} y$ \\ MELINA ELIZABETH ZULIANI ${ }^{2}$
}

\begin{abstract}
Summary: Effect of the nests of Acromyrmex lobicornis (Formicidae: Myrmicinae) on the decomposition of litter, in the "Monte" desert. Decomposition is a fundamental process of ecosystems. In arid and semiarid environments these processes slow down due to unfavorable climatic conditions for microbial activity so that organisms such as ants become key because in their nests they accumulate organic matter as a result of their foraging activities. Our objective was to evaluate the influence of the nests of Acromyrmex lobicornis on the decomposition process of the surrounding material. We buried 42 bags of litter in seven shrubs with nest and seven shrubs without nest (three bags per bush). At 21,35 and 55 days, the bags were removed, cleaned, weighed, dried and calcined. The rate of decomposition in nests of $A$. lobicornis was significantly lower than that of soils without anthills. The marked seasonality in the desert of Monte and the sterilization of the nest could be some of the causes.
\end{abstract}

Key words: Ants, arid zone, Acromyrmex lobicornis, Bulnesia retama, Formicidae, litter, Larrea cuneifolia, Myrmicinae, nests.

\begin{abstract}
Resumen: La descomposición es un proceso fundamental de los ecosistemas. En ambientes áridos y semiáridos, estos procesos se ralentizan debido a las condiciones climáticas desfavorables para la actividad microbiana, por lo que, organismos como las hormigas se vuelven claves ya que en sus nidos acumulan materia orgánica como consecuencia de su actividad de forrajeo. Nuestro objetivo fue evaluar la influencia de los nidos de Acromyrmex lobicornis sobre el proceso de descomposición del material circundante. Se enterraron 42 bolsas de hojarasca en 7 arbustos con y sin hormiguero (tres bolsas por arbusto). A los 21,35 y 55 días, las bolsas fueron retiradas, limpiadas, pesadas, secadas y calcinadas. La tasa de descomposición en nidos de A. lobicornis fue significativamente menor con respecto a la de suelos sin hormigueros. La marcada estacionalidad en el desierto del monte y la esterilización del nido podrían ser algunos de los causantes.
\end{abstract}

Palabras clave: Acromyrmex lobicornis, Bulnesia retama, Formicidae, hormigas, hojarasca, Larrea cuneifolia, Myrmicinae, nidos, zona árida.

\section{INTRODUCCIÓN}

La descomposición es uno de los procesos más importantes en los ecosistemas debido a su contribución de nutrientes al suelo, su repercusión en el carbono atmosférico global a través de la respiración microbiana y porque a través de la cadena de desintegradores fluye una cantidad significativa de energía que puede acumularse en el suelo como mantillo y humus (Prescott, 2005; Zhang et al., 2008).

La dinámica del proceso de descomposición ha sido ampliamente analizada en diferentes ecosistemas (Vitousek et al., 1994; Torres et al., 2005). En ambientes áridos, y en el desierto del monte en particular, los procesos de descomposición

\footnotetext{
${ }^{1}$ Instituto Argentino de Investigación de Zonas Áridas, Consejo Nacional de Investigaciones Científicas y Técnicas, Mendoza, CP 5500, Argentina.

2 Departamento de Análisis de Sistemas Complejos. Fundación Bariloche - Consejo Nacional de Investigaciones Científicas y Técnicas, Río Negro, Argentina.

*anavas@mendoza-conicet.gob.ar
} 
son muy lentos debido al bajo contenido de materia orgánica en los suelos como consecuencia de la baja biomasa vegetal, y condiciones climáticas desfavorables para la actividad microbiana (Bucher et al., 2003; Torres et al., 2005). El régimen escaso, variable e impredecible de las precipitaciones, presentes en estos sistemas, convierten a la disponibilidad de agua en uno de los factores más importantes que limitan el proceso de descomposición (Noy-Meir, 1973).

En estos ambientes, las hormigas cortadoras de hojas tendrían un rol clave en el proceso de descomposición (Farji-Brener \& Werenkraut, 2017) debido a su capacidad para generar y concentrar grandes cantidades de desechos orgánicos e incrementar el $\mathrm{N}$ mineral y otros nutrientes del suelo (Rico-Gray \& Oliveira, 2007; Bottinelli et al., 2015; Li et al., 2017). La contribución de materia orgánica de las hormigas cortadoras de hojas es de vital importancia en sitios donde la abundancia y la diversidad de materia orgánica es limitante, como en los sistemas desérticos (Farji-Brener, 2010). Los nidos de hormigas cortadoras son ricos en carbono orgánico y poseen mayor capacidad de retención de humedad que los suelos adyacentes (Farji-Brener \& Ghermandi, 2004; Abril \& Gómez, 2011). Estas hormigas tienen además la habilidad de modificar las propiedades físicas del suelo, aumentando la porosidad, la infiltración y la consistencia de los agregados del suelo (Davidson \& Morton, 1981; Eldridge \& Pickard, 1994). En consecuencia, ofrecen condiciones ideales para la reproducción y abundancia de la biota del suelo (Sousa-Souto et al., 2012).

En las zonas áridas de Argentina, Acromyrmex es un género de hormigas cortadoras, que se distribuye en todo el desierto del Monte, marcando los límites de distribución latitudinal y altitudinal de la tribu Attini en la Argentina. Este género se caracteriza por escindir material vegetal vivo que es utilizado como sustrato para el cultivo de un hongo simbiótico, que constituye el alimento directo de la colonia. Sus nidos son de gran tamaño y de larga duración, y depositan sus desechos, ricos en materia orgánica, en el exterior del mismo (Weber, 1972; Hölldobler \& Wilson, 1990). En los extremos de distribución, la única especie de hormiga cortadora que existe es Acromyrmex lobicornis Emery (Farji-Brener \& Ruggiero, 1994). Su hábitat incluye ambientes con grandes variaciones térmicas como montes, desiertos y estepas (Farji-Brener \& Ruggiero,
1994). Los nidos de A. lobicornis son más bien superficiales (Bollazzi et al., 2008), con basureros externos grandes y conspicuos (Farji-Brener, 2000) y usualmente con un domo externo que minimiza la variabilidad climática ambiental (Farji-Brener \& Sasal, 2003). Los nidos de A. lobicornis resultan así, sitios enriquecidos en nutrientes, con excelentes propiedades físico-químicas que le permitiría favorecer el ciclaje de nutrientes en estos sistemas (Farji-Brener \& Ghermandi, 2000).

En el desierto del Monte son escasos los trabajos que evalúan el proceso de descomposición, concentrándose la mayoría sobre el efecto del parche de vegetación como isla de fertilidad (Pucheta et al., 2006; Carretero et al., 2007; Carrera et al., 2009). Esto sumado a que un número de trabajos han demostrado un aumento de la fertilidad del suelo en nidos de hormigas debido al mayor aporte de nutrientes como consecuencia de sus actividades de forrajeo, (Culver \& Beattie, 1983; Dean \& Yeaton, 1993; McGinley et al., 1994; Petal \& Kusinska, 1994; Petal, 1998) y a que ninguno, para nuestro conocimiento, ha estudiado su efecto sobre la tasa de descomposición en el desierto del monte. El objetivo del presente trabajo fue conocer la tasa de descomposición de hojarasca de Larrea cuneifolia (CAB, 1800) en nidos de Acromyrmex lobicornis (Emery). Hipotetizamos que, los nidos de $A$. lobicornis incrementarán la tasa de descomposición de restos vegetales en el desierto del monte con respectos a sitios sin hormigueros.

\section{Materiales Y MÉtodos}

El área de estudio se localiza en Los Médanos Grandes, departamento Caucete, San Juan $\left(31^{\circ}\right.$ 43' Sur, $68^{\circ} 08^{\prime}$ Oeste). Pertenece a la Provincia Fitogeográfica del Monte (Cabrera, 1971). Tiene un clima seco y cálido, con inviernos fríos. La temperatura promedio para enero es de $25,1^{\circ} \mathrm{C}$ y para julio $7,7^{\circ} \mathrm{C}$. Las precipitaciones son escasas $\mathrm{y}$ estivales (diciembre a marzo), alcanzando un promedio de $100 \mathrm{~mm}$ anuales. La vegetación está dominada por arbustos como Bulnesia retama, Larrea divaricata, L. cuneifolia, Prosopis flexuosa, Capparis atamisquea y Senna aphylla, formando parches de cobertura densa dispersos en una matriz de suelo desnudo.

Para determinar la tasa de descomposición a campo, se seleccionaron 7 individuos de $B$. retama 


\section{A. L. Navas Romero et al. - Descomposición en nidos de Acromyrmex lobicornis}

que presentaron hormigueros de A. lobicornis bajo su dosel. Los controles consistieron en 7 individuos de $B$. retama sin la presencia de hormigueros. Durante la elección de cada réplica, se buscó que los hormigueros y arbustos seleccionados fueran similares en dimensiones (1,5-2 y 2-2,5 m diámetro respectivamente). Se utilizó el método de bolsas de hojarascas de Caldentey et al. (2001). El tamaño de las bolsas fue de $10 \times 10 \mathrm{~cm}$ y se construyeron usando telas mosquiteras plásticas con poros de 1 $\mathrm{mm}$, para evitar la pérdida de material y, a su vez, permitir la entrada de microorganismos. Utilizamos como material de descomposición hojas verdes de arbustos adyacentes de L. cuneifolia. Las hojas fueron recolectadas y separadas manualmente de la planta. Una vez homogeneizadas, se secaron a estufa a $60{ }^{\circ} \mathrm{C}$ hasta peso constante. Aproximadamente 1 $\mathrm{g}$ de hojas de L. cuneifolia se colocaron en cada bolsa. En agosto del 2016 (estación invernal), en el sitio de estudio, se ubicaron 3 bolsas con hojarasca a $5 \mathrm{~cm}$ de profundidad dentro del nido de hormigas (entrada del nido). Ubicamos cada bolsa de modo tal que toda su superficie permaneció en contacto con el nido. Realizamos el mismo procedimiento en los arbustos sin la presencia de nidos de hormigas ubicando 3 bolsas de hojarasca a $5 \mathrm{~cm}$ de profundidad. Las bolsas con hojarasca se ubicaron a $15 \mathrm{~cm}$ de distancia del tronco en cada una de los parches replicados para cada tratamiento ( 2 tratamientos $\times 7$ repeticiones $\times 3$ bolsas de hojarasca). Para estimar la pérdida de masa de la hojarasca, las 3 bolsas de cada parche se extrajeron sucesivamente, la primera a los 21 días (31 de agosto), la segunda a los 35 días (15 de septiembre) y la tercera a los 55 días (10 de octubre). En laboratorio, siguiendo a Pucheta et al. (2006) se limpiaron las bolsas con cepillos, para retirar restos de suelo, luego se extrajo la hojarasca de las bolsas, y se la dejo secar durante $48 \mathrm{~h} \mathrm{a} 60^{\circ} \mathrm{C}$. Cada muestra se pesó, y posteriormente se llevó a mufla a $400{ }^{\circ} \mathrm{C}$. Luego de $4 \mathrm{~h}$ las muestras se dejaron enfriar en desecador para evitar la absorción de agua del medio y se pesaron nuevamente.

Conociendo el peso de la biomasa colocada inicialmente y de la retirada al final del período, se evaluó el porcentaje de peso seco remanente (PSR) para cada tratamiento y tiempo de incubación. Se estimó las tasas de descomposición $(k)$ como las pendientes de las funciones exponenciales negativas para el PSR al aumentar el tiempo de incubación (Swift et al., 1979). Se evaluó si los PSR cumplían con los supuestos de una distribución normal. Las pendientes $(k)$ de las curvas de descomposición para los diferentes tratamientos se compararon por medio de una ANOVA de medidas repetidas. Utilizamos el programa Insfostat v2018 para realizar todos los análisis estadísticos y la confección de gráficos.

\section{Resultados}

Las bolsas de hojarasca extraídas bajo la copa de $B$. retama con la presencia de hormigueros presentaron un mayor PSR que las muestras extraídas en ausencia de hormigueros. A los 21 días de incubación PSR en suelos sin hormigueros fue 2,5 veces menor a la de suelo con hormigueros. A los 35 días la diferencia entre tratamientos fue de 2,1 veces. Al finalizar el experimento, luego de 55 días de incubación, el PSR en suelos sin hormigueros fue del $67 \%$ mientras que en suelos con hormigueros fue del $77 \%$ (Tabla 1 ).

La descomposición de la hojarasca se ajustó significativamente a funciones exponenciales negativas para parches de suelo con y sin hormigueros bajo el dosel de $B$. retama; y sus pendientes (constantes de decaimiento $k$ ) fueron diferentes significativamente (Figura 1). Observamos mayores tasas de descomposición de la hojarasca en el dosel de $B$. retama en suelos sin hormigueros que en suelos con hormigueros (ANOVA de medidas repetidas, $\mathrm{F}=5,08 ; \mathrm{p}<0,009$; $\mathrm{n}=21)$.

En cuanto la incidencia del tiempo de incubación sólo se encontraron diferencias significativas para el tratamiento sin hormigueros variando la tasa de descomposición significativamente entre los 21 y 55 días $(\mathrm{p}<0,032)$ y entre los 35 y 55 días $(\mathrm{p}<$ 0,039 ).

Tabla 1. Peso seco remanente (\%) para cada tratamiento (con nidos de $A$. Iobicornis y sin nido) y tiempo de incubación.

\begin{tabular}{|cccccccc|}
\hline & \multicolumn{3}{c}{ Con Hormiguero } & \multicolumn{3}{c|}{ Sin Hormiguero } \\
Tiempo & $\mathbf{n}$ & media & e.e. & $\mathbf{n}$ & media & e.e. \\
\hline 21 días & 7 & 91,36 & 2,22 & 7 & 78,02 & 2,93 \\
35 días & 7 & 86,79 & 2,81 & 7 & 77,44 & 3,53 \\
55 días & 7 & 77,75 & 4,28 & 7 & 67,97 & 3,98 \\
\hline
\end{tabular}



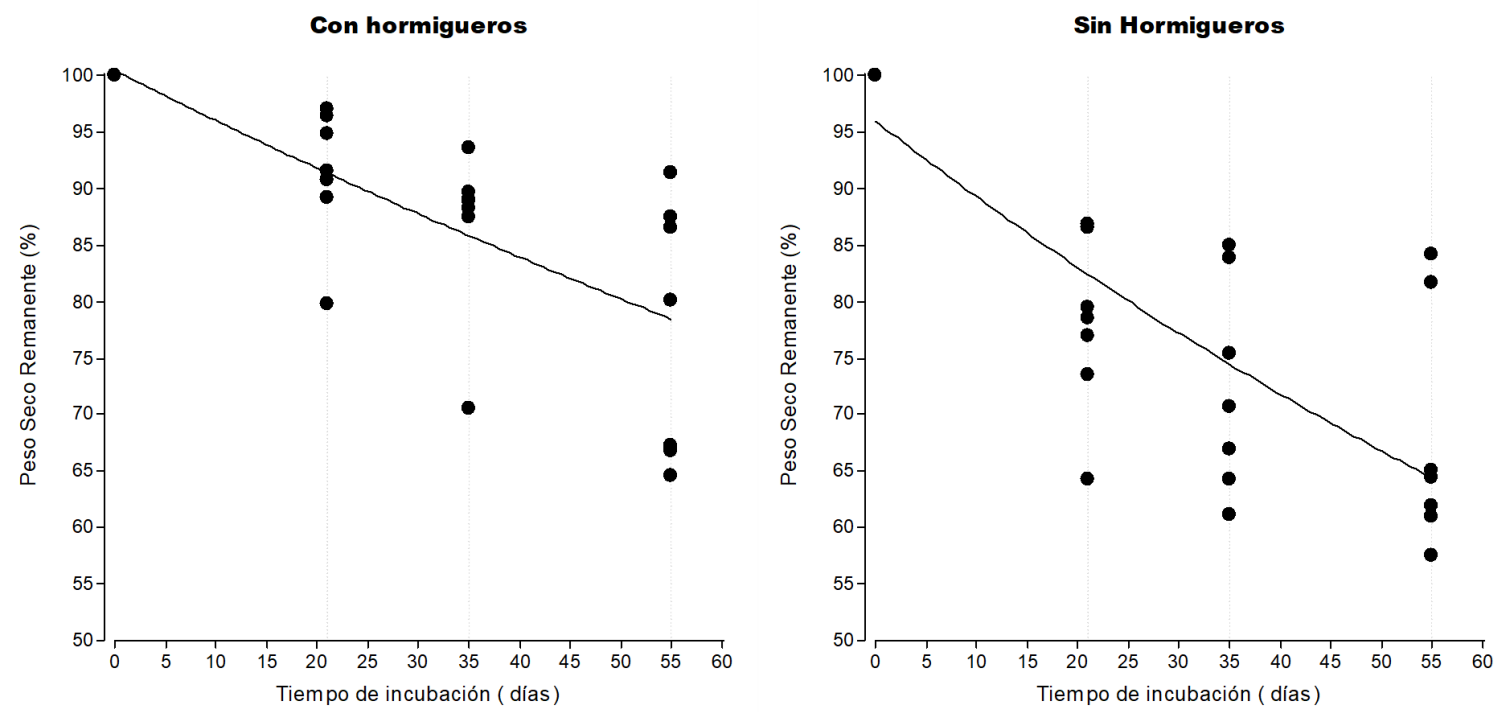

Fig. 1. Tasa de descomposición de la hojarasca enterrada (porcentaje de peso seco remanente) bajo el dosel de $B$. retama con la presencia o ausencia de hormigueros de $A$. lobicornis respectivamente, después de 21,35 y 55 días de incubación $(n=7)$. Las funciones negativas de decaimiento exponencial de la forma

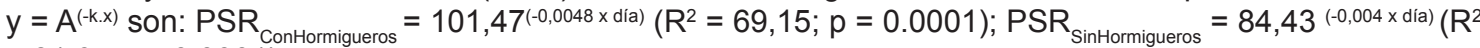
$=81,97 ; p<0,0001)$.

\section{Discusión}

Los nidos de A. lobicornis disminuyeron el proceso de descomposición de hojarasca de L. divaricata. La inhibición o estimulación de los procesos microbiológicos, por parte de las hormigas, depende del estado del proceso en el hábitat, el cual, a su vez, está fuertemente asociado con las condiciones climáticas y la calidad del alimento almacenado (Petal, 1978; Tadey \& FarjiBrener, 2007). La marcada estacionalidad presente en el desierto del Monte podría influir en que los nidos de A. lobicornis exista una menor tasa de descomposición. Según Gonzalez-Polo et al. (2004) para el desierto del monte, en diferentes épocas del año la actividad microbiana varía tanto dentro como fuera del hormiguero. En el período invernal (julio-noviembre) se registran valores bajos de descomposición y similares entre micrositios. En el siguiente período (noviembre-enero), el incremento en las temperaturas y precipitaciones llevaría a un aumento de la descomposición en ambos micrositios, siendo significativamente mayor en los nidos de hormigas. Resultados similares han sido obtenidos por Minner et al. (2009) para el desierto del monte, al analizar la dinámica de broza durante la estación de invierno en diferentes micrositios (parches de arbustos y suelo desnudo), donde no encontraron diferencias significativas, y por Vega Ávila et al. (2010) para el mismo sistema en la época invernal, al analizar la abundancia de microorganismo entre los distintos tipos de micrositios (parches de arbusto B. retama, $L$. divaricata, y suelo desnudo) y no encontrar variaciones ni grandes abundancias. Nuestro experimento estuvo comprendido en el período invernal-principio de primavera (agosto-octubre), durante esta época la actividad de forrajeo de las hormigas es casi nula, por lo que podría afectar directamente el contenido de materia orgánica, y a su vez la actividad microbiana. A esto debe sumarse las bajas temperaturas y humedades del suelo, y la disminución en la diversidad de la dieta de las hormigas que pareciera afectar directamente la actividad de los descomponedores (Fernández et al., 2014). Así la diferencia resultante en contenido de materia orgánica, humedad, disponibilidad de alimento, sumadas a bajas temperaturas podrían estar limitando el proceso de descomposición en los nidos de $A$. lobicornis.

Por otra parte $A$. lobicornis realiza una frecuente y necesaria esterilización del nido que podría estar 


\section{A. L. Navas Romero et al. - Descomposición en nidos de Acromyrmex lobicornis}

impactando en el proceso de descomposición. Las hormigas cortadoras de hojas tienen en general varios comportamientos y mecanismos químicos para garantizar una limpieza rigurosa del sustrato, protegerse a sí mismas y a su hongo mutualista, evitando la proliferación de agentes patógenos o de hongos competidores (Poulsen et al., 2006). Esto es esencial, ya que las condiciones óptimas para el hongo simbiótico, también son adecuados para muchas bacterias patógenas y hongos (Powell \& Stradling, 1986; Diehl-Fleig \& Labres, 1993; Fisher et al., 1996; Currie et al., 1999). Entre los mecanismos asociados a las hormigas encontramos: gestión de residuos y limpieza activa del nido (Wilson \& Hölldobler, 1980; Hölldobler \& Wilson, 1990; Bot et al., 2001; Currie \& Stuart, 2001; Hart \& Ratnieks, 2001), lamido con las partes bucales y cepillado con espinas de los protarsos (Perez \& Vázquez Moreno, 2004), producción de antibióticos por bacterias mutualistas (Currie et al., 1999, Currie, 2001), y la secreción de sustancias antifúngicas y antibacterianas por la glándula metapleural. Esta última sería la más importante de todas y se encuentra presente en el género Acromyrmex (Hölldobler \& Engel-Siegel, 1984). La glándula metapleural ha sido implicada como una fuente importante de sustancias de defensa e higiénica tanto para el individuo como para la colonia (Beattie et al., 1985, 1986, a, b). Estas glándulas producen una secreción que contiene más de 20 compuestos diferentes y se sabe que estos tienen propiedades bactericidas y fúngicas (Do Nascimento et al., 1996; Ortius-Lechner et al., 2000; Bot et al., 2002; Poulsen et al., 2002). La importancia y el rol como esterilizantes de estas glándulas ha sido ampliamente estudiado (Veal et al., 1992; Ortius-Lechner et al., 2000; Poulsen et al., 2006). Así las hormigas mantienen, en la medida de lo posible, un entorno libre de competencia para el hongo mutualista eliminando constantemente otros hongos e infecciones microbianas (Bass \& Cherrett, 1994), cumpliendo un rol controversial en la desactivación de los procesos de descomposición.

La diferencia en el porcentaje seco remanente entre tratamientos fue marcada al inicio del experimento y disminuyó progresivamente con el tiempo de incubación. Esta diferencia entre ambos, es atribuible a los factores abióticos que dominan la primera etapa del proceso de descomposición (primeros 30 días), como por ejemplo el contenido de humedad, la exposición a radiación y variaciones térmicas (Camilleri \& Ribi, 1986). Estos aparentemente se verían disminuidos en los nidos de A. lobicornis, que por sus características, reducirían la incidencia de la radiación y elevarían la hidrofobia impidiendo el mayor drenaje de agua (Cammeraat et al., 2002). Los hormigueros requerirían pulsos largos para pasar el límite de hidrofobia, el cual es muy alto al inicio, por la elevada carga de material orgánico en el exterior (Cammeraat et al., 2002; Wills \& Landis, 2017), lo cual no sucede en el suelo descubierto (arenoso y sin mantillo). Hacia el final del experimento las diferencias se atenuaron, en esta etapa la descomposición es más lenta y mediada por poblaciones microbianas y fúngicas que requieren para iniciarse de mayor tiempo de incubación y condiciones óptimas rigurosas, las cuales también parecen ser desfavorables en los nidos de A. lobicornis (Farji-Brener et al., 1995).

Así los suelos arenosos desérticos bajo arbustos de $B$. retama tendrían un mayor potencial de descomposición de hojarasca que los nidos de las hormigas cortadoras A. lobicornis en el periodo evaluado. Los nidos de hormigas jugarían un rol contradictorio en el ciclado de nutrientes, debido a su capacidad de movilizar grandes volúmenes de materia orgánica al interior de los hormigueros, afectando así el funcionamiento del ecosistema del Monte. La baja tasa de descomposición en los nidos de $A$. lobicornis podría generar un aumento en la retención de los nutrientes y una disminución en la cantidad de nutrientes disponibles para los productores primarios. Estos cambios afectarían procesos asociados como disminución en la germinación y presencia de plántulas, menor riqueza de especies y densidad de plantas (Carson \& Peterson, 1990; Jensen \& Meyer, 2001). Sin embargo, dada la complejidad de los sistemas naturales, y del rol que juegan las hormigas cortadoras en los procesos ecosistémicos (Farji-Brener et al., 2017), son necesarios estudios a una escala de tiempo mayor.

\section{Agradecimientos}

A la cátedra de Ecología II de la UNSJ, por guiarnos durante la realización del trabajo. A la Facultad de Ingeniería por su asistencia en el trabajo de laboratorio. 


\section{Bibliografía}

BASS, M. \& J. CHERRETT. 1994. The role of leafcutting ant workers (Hymenoptera: Formicidae) in fungus garden maintenance. Ecol. Entomol. 19: 215-220.

BEATtie, A., C. TURNBUlL, T. HOUGH \& R. KNOX. 1985. The vulnerability of pollen and fungal spores to ant secretion: evidence and some evolutionary implications. Amer. J. Bot. 72: 606-614.

BEATTIE, A., C. TURNBUlL, T. HOUGH \& R. KNOX. 1986. Antibiotic production: a possible function for the metapleural glands of ants Myrmecia nigriscapa, Hymenoptera: Formicidae. Ann. Entomol. Soc. Am. 79: 448-450.

BOLLAZZI, M., J. KRONENBITTER \& F. ROCES. 2008. Soil temperature, digging behaviour, and the adaptive value of nest depth in South American species of Acromyrmex leaf-cutting ants. Oecologia 158: 165-175.

BOT, A., D. ORTIUS-LECHNER, K. FINSTER, R. MAILE \& J. BOOMSMA. 2002. Variable sensitivity of fungi and bacteria to compounds produced by the metapleural glands of leaf-cutting ants. Insectes Soc. 49: 363-370.

BOT, A., M. OBERMAYER, B. HÖLLDOBLER \& J. BOOMSMA. 2001. Functional morphology of the metapleural gland in the leaf-cutting ant Acromyrmex octospinosus. Insectes Soc. 48: 6366.

BOtTINELli, N., P. JOUQUET, Y. CAPOWIEZ, P. PODWOJEWSKI, M. GRIMALDI \& X. PENG. 2015. Why is the influence of soil macrofauna on soil structure only considered by soil ecologists? Soil Tillage Res. 146: 118-124.

BUCHER, E., P. TORRES \& A. ABRIL. 2003. Litter quality and litter removal by the native fauna in the western Chaco woodland of Argentina. J. Trop. Ecol. 19: 337-341.

CABRERA, A. 1971. Fitogeografía de la República Argentina. Bol. Soc. Argent. Bot. 14: 1-42.

CALDENTEY, J., M. IBARRA \& J. HERNÁNDEZ. 2001. Litter fluxes and decomposition in Nothofagus pumilio stands in the region of Magallanes, Chile. Forest Ecol. Manag. 148: 145-157.

CAMILlERI, J. \& G. RIBI. 1986. Leaching of dissolved organic carbon (DOC) from dead leaves, formation of flakes from DOC, and feeding on flakes by crustaceans in mangroves. Mar. Biol. 91: 337-344.

CAMMERAAT, L., S. WILLOTT, S. COMPTON \& L. INCOLL. 2002. The effects of ants' nests on the physical, chemical and hydrological properties of a rangeland soil in semi-arid Spain. Geoderma 105: 1-20.
CARRERA, A., M. MAZZARINO, M. BERTILLER, H. DEL VALLE \& E. MARTINEZ CARRETERO. 2009. Plant impacts on nitrogen and carbon cycling in the Monte phytogeographical province, Argentina. J. Arid Environ. 73: 192-201.

CARSON, W. \& C. PETERSON. 1990. The role of litter in an old-field community: impact of litter quantity in different seasons on plant species richness and abundance. Oecologia 85: 8-13.

CULVER, D. \& A. BEATTIE. 1983. Effects of ant mounds on soil chemistry and vegetation patterns in a Colorado Montane Meadow. Ecology 64: 485-492.

CURRIE, C. \& A. STUART. 2001. Weeding and grooming of pathogens in agriculture by ants. Proc. R. Soc. London, Ser. B 268: 1033-1039.

CURRIE, C., U. MUELLER \& D. MALLOCH. 1999. The agricultural pathology of ant fungus gardens. PNAS 96: 7998-8002.

DAVIDSON, D. \& S. MORTON. 1981. Competition for dispersal in ant-dispersed plants. Science 213: 1259- 1261.

DEAN, W. \& R. YEATON. 1993. The effects of harvester ant Messor capensis nest-mounds on the physical and chemical properties of soils in the Southern Karoo, South Africa. J. Arid Environ. 25: 249-260.

DIEHL-FLEIG, E. \& M. LABRES. 1993. Fungi isolated from leaf-cutting ants Atta sexdens piriventris and Acromyrmex heyeri (Hymenoptera: Formicidae): Mucor effects on Beauveria bassiana entomopathogen. Ciênc. Cult. 45: 142-144.

DO NASCIMENTO, R., E. SCHOETERS, E. MORGAN, J. BILLEN \& D. STRADLING. 1996. Chemistry of metapleural gland secretions of three attine ants, Atta sexdens rubropilosa, Atta cephalotes, and Acromyrmex octospinosus (Hymenoptera: Formicidae). J. Chem. Ecol. 22: 987-1000.

ELDRIDGE, D. \& J. PICKARD. 1994. Effects of ants on sandy soils in semi-arid eastern Australia: II. Relocation of nest entrances and consequences for bioturbation. Aust. J. Soil Res. $\quad 32:$ 323-333.

FARJI-BRENER, A. 2000. Leaf-cutting ant nests in temperate environments: mounds, mound damages and nest mortality rate in Acromyrmex lobicornis. Stud. Neotrop. Fauna Environ. 35: 131-138.

FARJI-BRENER, A. 2010. Leaf-cutting ant nests and soil biota abundance in a semi-arid steppe of NW Patagonia. Sociobiology 56: 549-557.

FARJI-BRENER, A. \& A. RUGGIERO. 1994. Leafcutting ants (Atta and Acromyrmex) inhabiting Argentina: patterns in species richness and geographical range sizes. J. Biogeogr. 21: 391-399.

FARJI-BRENER, A. \& J. SILVA. 1995. Leaf-cutting ant nests and soil fertility in a well-drained savanna in western Venezuela. Biotropica 27: 250-254. 


\section{A. L. Navas Romero et al. - Descomposición en nidos de Acromyrmex lobicornis}

FARJI-BRENER, A. \& L. GHERMANDI. 2000. Influence of nests of leaf-cutting ants on plant species diversity in road verges of northern Patagonia. J. Veg. Sci. 11: 453-460.

FARJI-BRENER, A. \& L. GHERMANDI. 2004. Seedling recruitment in a semi-arid Patagonian steppe: Facilitative effects of refuse dumps of leaf-cutting ants. J. Veg. Sci. 15: 823-830.

FARJI-BRENER, A. \& V. WERENKRAUT. 2017. The effects of ant nests on soil fertility and plant performance: a meta-analysis. J. Anim. Ecol. 86: 866-877.

FARJI-BRENER, A. \& Y. SASAL. 2003. Is dump material an effective small-scale deterrent to herbivory by leafcutting ants? Ecoscience 10: 151-154.

FERRARI, L., F. DE LA TORRE, S. DEMICHELIS, M. GARCÍA \& A. SALIBIÁN. 2014. Ecotoxicological assessment for receiving waters with the premetamorphic tadpoles acute assay. Chemosphere 59: 567-575.

FISHER, P., D. STRADLING, B. SUTTON \& L. PETRINI. 1996. Microfungi in the fungus gardens of the leaf-cutting ant Atta cephalotes: A preliminary study. Mycol. Res. 100: 541-546.

GONZALEZ-POLO, M., P. FOLGARAIT \& A. MARTÍNEZ. 2004. Evaluación estacional del efecto de los nidos de Camponotus punctulatus sobre la biomasa y la actividad microbiana en una pastura subtropical de Argentina. Ecol. Aust. 14: 149-163.

HART, A. \& F. RATNIEKS. 2001. Task partitioning, division of labour and nest compartmentalisation collectively isolate hazardous waste in the leafcutting ant Atta cephalotes. Behav. Ecol. Sociobiol. 49: 387-392.

HOLLDOBLER, B. \& H. ENGEL-SIEGEL. 1984. On the metapleural glands of ants. Psyche (Stuttg). 91: 201-224.

HÖLLDOBLER, B. \& E. WILSON. 1990. The Ants. 1st ed. Springer-Verlag, Berlin-Heidelberg.

JENSEN, K. \& C. MEYER. 2001. Effects of light competition and litter on the performance of Viola palustris and on species composition and diversity of an abandoned fen meadow. Plant Ecol. 155: 169-181.

LI, T., M. SHAO \& Y. JIA. 2017. Effects of activities of ants (Camponotus japonicus) on soil moisture cannot be neglected in the northern Loess Plateau. Agric. Ecosyst. Environ. 239: 182-187.

MARTINEZ CARRETERO, E., A. DALMASSO \& S. TRIONE. 2007. Carbon Storage in Larrea divaricata and L. cuneifolia (Zygophyllaceae) in Drylands of Central-Western Argentina. Arid Land Res. Manag. 21: 273-285.

MCGINLEY, M., S. DHILLION \& J. NEUMANN. 1994. Environmental heterogeneity and seedling establishment: ant-plant-microbe interactions. Funct. Ecol. 8: 607-615.
MINER, A., J. ALVAREZ, P. VILLAGRA \& J. ARANIBAR. 2010. Dinámica temporal de la masa de broza en distintos microhábitats del desierto del Monte Central (Mendoza, Argentina). Rev. Fac. Cienc. Agrar. 42: 55-69.

NOY-MEIR, I. 1973. Desert ecosystems: environment and producers. Annu. Rev. Ecol. Syst. 4: 25-51.

ORTIUS-LECHNER, D., R. MAILE, E. DAVID MORGAN \& J. BOOMSMA. 2000. Metapleural gland secretion of the leaf-cutter ant Acromyrmex octospinosus: New compounds and their functional significance. J. Chem. Ecol. 26: 1667-1683.

PEREZ, N. \& L. VAZQUEZ MORENO. 2004. Manejo ecológico de plagas. En: PEREZ, N. (ed.), Transformando el Campo cubano. Avances de la agricultura sostenible, pp. 191-223. La Habana. Cuba: Universidad Agraria de La Habana. Centro de Estudios de Desarrollo Agrario y Rural.

PETAL, J. 1978. The role of ants in ecosystems. In: BRIAN M. V. (Ed.), Production ecology of ants and termites, pp. 293-325. Cambridge University Press, London.

PETAL, J. 1998. The influence of ants on carbon and nitrogen mineralization in drained fen soils. Appl. Soil Ecol. 9: 271-275.

PETAL, J. \& A. KUSINSKA. 1994. Fractional composition of organic-matter in the soil of anthills and of the environment of meadows. Pedobiologia 38: 493-501.

POULSEN, M., A. BOT, M. NIELSEN \& J. BOOMSMA. 2002. Experimental evidence for the costs and hygienic significance of the antibiotic metapleural gland secretion in leaf-cutting ants. Behav. Ecol. Sociobiol. 52: 151-157.

POULSEN, M., W. HUGHES \& J. BOOMSMA. 2006. Differential resistance and the importance of antibiotic production in Acromyrmex echinatior leaf-cutting ant castes towards the entomopathogenic fungus Aspergillus nomius. Insect Soc. 53: 349-355.

POWELL, R. \& D. STRADLING. 1986. Factors influencing the growth of Attamyces bromatificus, a symbiont of attine ants. T. Brit. Mycol. Soc. 87: 205-213.

PRESCOTT, C. 2005. Do rates of litter decomposition tell us anything we really need to know?. For. Ecol. Manage. 220: 66-74.

PUCHETA, E., M. LlANOS, C. MEGLiOLi, M. GAVIORNO, M. RUIZ \& C. PARERA. 2006. Litter decomposition in a sandy Monte desert of western Argentina: influences of vegetation patches and summer rainfall. Austral Ecol. 31: 808-816.

RICO-GRAY, V. \& P. OLIVEIRA. 2007. The Ecology and Evolution of Ant-Plant Interactions. University of Chicago Press, Chicago. 
SOUSA-SOUTO, L., D. SANTOS, B. AMBROGI, M. SANTOS, M. GUERRA \& E. PEREIRA-FILHO. 2012. Increased $\mathrm{CO}_{2}$ emission and organic matter decomposition by leaf-cutting ant nests in a coastal environment. Soil Biol. Biochem. 44: 21-25.

TADEY, M. \& A. FARJI-BRENER. 2007. Indirect effects of exotic grazers: Livestock decreases the nutrient content of refuse dumps of leaf-cutting ants through vegetation impoverishment. J. Appl. Ecol. 44: 1209-1218.

TORRES, P., A. ABRIL \& E. BUCHER. 2005. Microbial succession in litter decomposition in the semi-arid Chaco woodland. Soil Biol. Biochem. 37: 49-54.

VEAL, D., J. TRIMBLE \& A. BEATTIE. 1992. Antimicrobial properties of secretions from the metapleural glands of Myrmecia gulosa (the Australian Bull Ant). J. Appl. Bacteriol. 72: 188194.

VEGA ÁVIlA, Á., M. TORO, M. BAIGORI, L. FERNÁNDEZ \& F. VÁZQUEZ. 2010. Influencia de la vegetación en la variación espacial de la abundancia de microorganismos en el desierto del Monte, San Juan, Argentina. Ecol. Aus. 20: 247-256.
VITOUSEK, P., D. TURNER, W. PARTON \& R. SANFORD. 1994. Litter decomposition on the mauna loa environmental matrix Hawaii' i: Patterns, mechanisms, and models. Ecology 75: 418-429.

WEBER, N. 1972. The fungus culturing behavior of ants. Am. Zool. 12: 577-587.

WILLS, B. \& D. LANDIS. 2017. The role of ants in north temperate grasslands: a review. Oecologia 186: $1-16$.

WILSON, E. \& B. HOLLDOBBLER. 1980. Sex differences in cooperative silk spinning by weaver ant larvae. PNAS 77: 2343-2347.

ZHANG, D., D. HUI, Y. LUO \& G. ZHOU. 2008. Rates of litter decomposition in terrestrial ecosystems: global patterns and controlling factors. J. Plant Ecol. 1: 85-93.

Recibido el 25 de junio de 2018, aceptado el 24 de septiembre de 2018. Editor: Omar Varela. 